measures (area under curve (AUC)) were anchored around known anatomical landmarks as identified by compartmental $\mathrm{pH}$ changes. 60-minute epochs were used to quantify antral, duodenal, ileal, cae$\mathrm{cal}$ and distal colonic contractility. The maximum and minimum $\mathrm{pH}$ was measured either side of the ileo-caecal junction. All data are presented as means $( \pm 95 \% \mathrm{CI})$.

Results No differences were seen in any of the motility parameters, compartmental transit times or maximal ileal $\mathrm{pH}$ between the two groups. Minimum caecal $\mathrm{pH}$ was significantly lower in patients compared to control ( $\mathrm{Px}=5.14 \pm 0.14 \mathrm{v} \mathrm{Cx}=6.12 \pm 0.16, \mathrm{p}<0.0001)$. The $95 \%$ CI for maximum $\mathrm{pH}$ drop across the ICJ in health was 1.65 units. There was a significant correlation between caecal $\mathrm{pH}$ and caecal contractility $(r=0.498, p=0.05)$.

Conclusion In this study, we have shown that patients with lower abdominal symptoms typically associated with, but not limited to, conditions such as irritable bowel syndrome (IBS) have a significantly lower caecal $\mathrm{pH}$ compared to controls. This low $\mathrm{pH}$ environment is maintained by fermentation and subsequent SCFA production. SCFA have been shown to inhibit colonic motility in-vitro and contractility as measured by the WMC was correlated with caecal $\mathrm{pH}$. With the recent success of anti-biotic therapy and low fermentable diets in the treatment of lower bowel symptoms in IBS, measurement of caecal $\mathrm{pH}$ using the WMC provides an objective and quantifiable biomarker of fermentation. This may be used to sub-classify patients with a broad spectrum of GI disorders and identify those that may benefit most from antibiotic, probiotic and dietary interventions providing novel insights into the pathophysiological mechanisms of lower GI symptoms.

Disclosure of Interest A. Hobson Paid Instructor for: Given Imaging, S. Mohammed: None Declared, G. Dukes Employee of: GSK, M. Scott: None Declared

\section{PWE-010 BIOFEEDBACK-A SIMPLE AND EFFECTIVE WAY OF MANAGING RECTAL EVACUATORY DYSFUNCTION SECONDARY TO PELVIC FLOOR DYSSYNERGIA AND RECTAL HYPOSENSITIVITY}

doi:10.1136/gutjnl-2013-304907.299

1." C Rimmer, 'K Stackhouse, 'N Cruickshank, 'K Gill. 'FINCH Unit, Sandwell and West Birmingham NHS Trust, Birmingham, UK

Introduction Biofeedback is safe and effective in the management of rectal evacuatory dysfunction (RED), but there is limited data on medium to long-term follow-up. This study evaluated the effectiveness of biofeedback in the medium-term for patients with RED secondary to pelvic floor dyssynergia (PFD) and rectal hyposensitivity (RH).

Methods Prospective data was collected from 2010 -2013 of 81 consecutive patients who underwent specialist nurse-led verbal biofeedback therapy in the form of rectal sensory re-training or balloon expulsion for PFD or RH. The primary outcome measure was patient satisfaction with their symptom improvement, assessed using a visual analogue scale (Likert scale $0-10$ ). Secondary outcome measures were complete spontaneous bowel movements (CSBMs)/ week, time to defecation-assessed using bowel diaries, and KESS/ SF-36 quality of life questionnaire scores. All discharged patients received telephone follow-up.

Results $85 \%$ patients met the primary outcome measure, with the Likert score improving [mean baseline $3.2(1-7)$ vs. post-biofeedback 7.6 (5-9) $\mathrm{p}<0.001$ ]. Improvements were seen in CSBMs/week [mean baseline: $3.0(1-14)$ vs. post-biofeedback: $6.9(1-13) \mathrm{P}<0.001]$ and time taken to defecate in minutes [mean baseline: 18.7 (5-60) vs. post-biofeedback: 8.7(5-30) $\mathrm{P}<0.001]$. Significant improvements were seen in KESS [mean baseline 13.1 (9-18) vs. post-biofeedback $4.9(0-16)$ p $<0.001]$ and SF-36 scores. Mean number of biofeedback treatments received was four (1-6). $89 \%$ of patients discharged still meet the primary endpoint at a mean follow-up of 19 months (range 7-36).

Conclusion Biofeedback has a key role to play in the management of rectal evacuatory dysfunction secondary to pelvic floor dyssynergia and rectal hyposensitivity, with the improvement being maintained in the medium-term.

Disclosure of Interest None Declared.

\section{PWE-011 CHARTING THE VOLATILE ORGANIC METABOLOME (VOM): A COMPARISON OF MURINE AND HUMAN FAECES}

doi:10.1136/gutjnl-2013-304907.300

1.* D Sawbridge, ' $\mathrm{S}$ Reade, ${ }^{\top} \mathrm{T}$ Khalid, ${ }^{1} \mathrm{C}$ Probert. 'Gastroenterology, University of Liverpool, Liverpool, UK

Introduction The metabolome is important in the development of IBD and colorectal cancer. It may be studied using gas chromatography mass spectrometry (GCMS) or electronic noses that sniff the volatile organic compounds, which comprise a large portion of the metabolome. However the healthy volatile organic metabolites (VOMs) of mice remain uncharted, nor have mouse models been validated for use in comparative studies of VOMs with humans.

Methods We collected serial samples from healthy C57 BL/6 mice and a healthy human, as part of our method development for VOM studies in colitis and colorectal cancer. The samples were enclosed in glass vials and the VOMs extracted from the headspace gas. VOMs were analysed using GCMS and the resultant fragmentation patterns compared to the NIST database for compound identification. VOMs from murine and human samples were compared. The consistency of mouse and human samples over time and between individuals was also examined by listing the rank position in quintiles over serial samples.

Results 60 individual VOMs were identified from mice and 87 in the human, with a $22 \%$ correlation between the two. Most notable in the murine sample was the high concentration of simple organic molecules such as hexane and short chain aldehydes. Several compounds were found in the murine sample that had not been seen in human samples and probably reflect urine contamination from incontinent mice. The greatest concentration of VOMs in the mouse were: hexanal, 2.2.4.6.6-pentamethyl-heptane and pentanal. By comparison, in the human, complex cycloalkenes and benzene-based compounds, such as 1-methyl-4-(1-methylethylidene)-cyclohexene, were in abundance. Chief compounds in the human were: D Limonene, 2-methyl phenol and indole. Several molecules were prominent in both mouse and human, such as acetone, short chain organic acids including hexanoic, pentanoic and butanoic acids and D-limonene. The more simple compounds likely reflect a combination of the metabolic effluvia of common intestinal microbiota and mammalian physiology. The position of VOMs in each quartile was found to be consistent with $86 \%$ recurrence in the first quartile, $71 \%$ in the last quartile.

Conclusion This is the first description of the VOC metabolome of $\mathrm{C} 57 \mathrm{BL} / 6$ mice. A comparison with human samples shows there is a low but notable correlation between the two species. Many of the VOCs present are by-products of microbial and cellular metabolism. The difference in number and complexity of the VOCs found in the human, compared with the mouse, may reflect varied diet and a more complex intestinal microbiome. Such data will allow calibration of future studies of colonic disease in humans and through the induction of colonic disease in mice.

Disclosure of Interest None Declared.

\section{PWE-012 ASSESSMENT OF BACTERIAL DIVERSITY IN COLORECTAL ADENOMATOUS POLYPS}

doi:10.1136/gutjnl-2013-304907.301

1.* $\mathrm{G}$ Hold, ${ }^{1} \mathrm{M}$ Hope, ${ }^{1} \mathrm{P}$ Lochhead, ${ }^{2} \mathrm{C}$ Meharg, ${ }^{1} \mathrm{~S}$ Berry, ${ }^{1} \mathrm{E}$ El-Omar. 'Division of Applied Medicine, Aberdeen University, Aberdeen; ${ }^{2}$ Queens University, Belfast, UK 